\title{
Model-free decomposition of transient absorption spectra into components with time-dependent shape
}

\author{
B. Lang ${ }^{1}$, A. Rosspeintner ${ }^{1}$, G. Angulo ${ }^{2}$ and E. Vauthey ${ }^{1}$ \\ ${ }^{1}$ Department of Physical Chemistry, University of Geneva, 30 quai Ernest Ansermet, CH-1211 Geneva 4, Switzerland \\ ${ }^{2}$ Institute of Physical Chemistry, Polish Academy of Sciences, 01-224 Warsaw, Poland
}

\begin{abstract}
Disentangling overlapping spectral signatures with time-dependent shape is performed using additional information contained in ultrafast transient absorption data, without applying any assumption on the underlying kinetics.
\end{abstract}

Electronic transient absorption (TA) spectroscopy is used throughout the field of ultrafast spectroscopy to monitor transient populations evolving on excited state potential or free energy surfaces in real-time. Broad and overlapping spectral features of the various contributing states and species constitute a commonly encountered problem.

As long as these spectral signatures do not change their shape over time, a well-developed machinery of chemometric procedures using mathematical tools such as singular value decomposition [1] or global and target analysis [2] can be applied to disentangle the contributions and to extract their kinetics. Such methods typically need as input some information about the underlying kinetics, e.g. a system of linear or nonlinear rate equations. Even more importantly, they rely on a description based on a separation of the spectral and temporal variables. Starting from a matrix containing columns of pixel values measured by the TA spectrometer as a function of time delay, these methods essentially perform a decomposition into a product of two matrices, one containing exclusively spectral information and the other exclusively kinetic information.

However, on the ultrafast time scale, population dynamics are most often accompanied by processes such as vibrational relaxation and solvent reorganization which typically induce lineshape changes and shifts of the spectral features during the first tens of picoseconds. Thus, in a description based on the underlying physical process, a separation of the spectral and temporal variables is not possible. A purely numerical description of the experimental data can of course always be achieved when using a sufficient number of components with corresponding kinetics [3, 4] However, the resulting values cannot be associated to specific species or processes. When the bands of all species are known, a decomposition is of course always possible. Otherwise, additional information has to be used to determine unknown spectral signatures. In this contribution we show a couple of examples how to obtain such information from the experimental data without applying any specific model to the underlying kinetics.
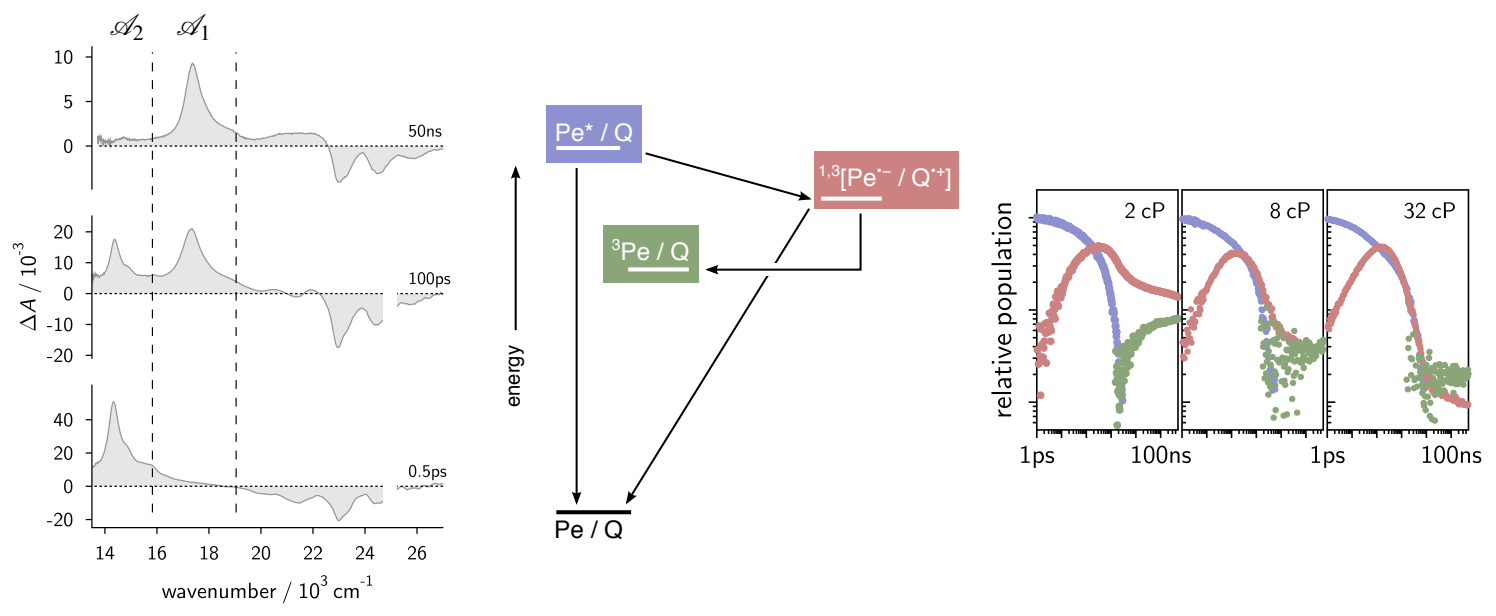

Figure 1: Selected transient spectra of photoinduced electron transfer between photoexcited perylene $(\mathrm{Pe})$ and $\mathrm{N}, \mathrm{N}$-dimethylaniline (left panel), reaction scheme (middle panel, $\mathrm{Q}=\mathrm{DMA}$ ) and extracted concentration kinetics (right panel), blue ${ }^{1} \mathrm{Pe}^{*}, \mathrm{red} \mathrm{Pe}^{-}$and green ${ }^{3}$ Pe population.

The left panel of fig. 1 shows a series of transient absorption spectra of perylene (Pe) in acetonitrile (ACN) in the presence of 0.3 mol $\mathrm{N}, \mathrm{N}$-dimethylaniline (DMA) at different probing times. The spectrum at $500 \mathrm{fs}$ shows exclusively features of the excited Pe molecule upon photoexcitation with an ultrafast laser pulse at $400 \mathrm{~nm}$. Its excited state absorption (ESA) is located below $18 \mathrm{kK}$, the stimulated emission (SE) between $18 \mathrm{kK}$ and $23 \mathrm{kK}$ and the the ground state bleach above $23 \mathrm{kK}$. During the diffusion assisted encounter with 
DMA the ${ }^{1} \mathrm{Pe}^{*}$ state is quenched by electron transfer. In the spectrum at $100 \mathrm{ps}$ the peak at about $17.5 \mathrm{kK}$ can be attributed to the Pe radical anion. Note that the contribution of $\mathrm{DMA}^{+}$is structureless and negligibly small compared to that of the Pe anion features. As indicated in the scheme in the middle panel of fig. 1, the ion pair can recombine into a neutral pair or diffusively separate to free ions. During a diffusive re-encounter, also recombination to a neutral pair in the triplet state is possible. The signature of the latter is located at $20-22 \mathrm{kK}$.

When extracting the kinetics of the species contributing to the spectra shown in fig. 1, one is faced with the problem that the bands of the ${ }^{1} \mathrm{Pe}^{*}$ ESA and SE exhibit spectral changes due to vibrational relaxation. Since these changes are present also without quencher, they can be determined in a separate experiment. A more detailed analysis shows that spectral dynamics are also present on the band of the Pe anion. Note that even in the absence of such spectral dynamics, a decomposition based on steady state absorption spectra obtained from electrochemically or chemically generated ions can be difficult due to static shifts induced by the presence of counter ions of different nature.

The sum of the signals due to ${ }^{1} \mathrm{Pe}^{*}$ and the $\mathrm{Pe}^{-}$radical cation can be written as the ${ }^{1} \mathrm{Pe}^{*}$ signal in the absence of quencher times a quenching factor $q_{\mathrm{S}}(c, t)$ where $c$ indicates the concentration of DMA

$$
\Delta A(c, t, v)=\Delta A_{\mathrm{S}}(c, t, v)+\Delta A_{\mathrm{prod}}(c, t, v)=q_{\mathrm{s}}(c, t) \cdot \Delta A(0, t, v)+\Delta A_{\mathrm{prod}}(c, t, v) .
$$

Taking into account that the strength of the electronic transition dipoles at the origin of the spectrum of Pe $\mathrm{e}^{+}$do not substantially change during vibrational relaxation, one can assume that the areas under the different spectral regions do not change and keep a constant ratio amongst each other

$$
\underbrace{\int_{2} \Delta A_{\text {prod }}(c, t, v) \mathrm{d} v}_{:=\mathscr{A}_{2}(c, t)}=\alpha \underbrace{\int_{1} \Delta A_{\text {prod }}(c, t, v) \mathrm{d} v}_{:=\mathscr{A}_{1}(c, t)} .
$$

The areas $\mathscr{A}_{1}(c, t)$ and $\mathscr{A}_{2}(c, t)$ are indicated by dashed lines in fig. 1, left panel. Inserting (1) into (2) yields the quenching factor

$$
q_{\mathrm{S}}(c, t)=\frac{\alpha \mathscr{A}_{1}(c, t)-\mathscr{\mathscr { A }}_{2}(c, t)}{\alpha \mathscr{A}_{1}(0, t)-\mathscr{\mathscr { A }}_{2}(0, t)} .
$$

Evaluating the obtained $q_{\mathrm{S}}(c, t)$ leads to the quenching dynamics and thus, the contribution of the ${ }^{1} \mathrm{Pe}^{*}$ state can be removed. The remaining product signal can be further decomposed into the contributions of the Pe anion and the Pe triplet state following the same procedure. The resulting concentration kinetics are shown in fig. 1, right panel, which can be used to quantitatively test reaction models of diffusion controlled electron transfer quenching [5].

The so-far described procedure leads to kinetic traces of the spectroscopic signatures. Of more interest are the underlying population dynamics. The link is provided by the corresponding extinction coefficients which can be obtained from the disentangled spectra by comparing the ground state bleach with the corresponding steady state absorption spectrum. However, in many cases, as the present one, there is an ESA to be found on top of the ground state bleach preventing a direct comparison. Wilcken et al. have shown how to use the second order derivative of the band shape to disentangle both contributions [6]. The basic idea is that the measured spectrum is decomposed such that the known features of the steady state absorption are present only in one of the two components. Knowing the extinction coefficient of the $S_{0} \rightarrow S_{1}$ transition, all contributing extinction coefficients from Pe in the $S_{1}$ state can be determined. With that information and the kinetics at hand, all other extinction coefficients can be determined, permitting an often omitted cross-check of kinetic models applied for interpreting the experimental data.

Which one of the two described procedures is the method of choice in a particular case depends on the spectral features actually being found in the experimental data. Obviously, the method relying on the band shape works best when the known compound spectrum exhibits pronounced features and the unknown is rather featureless, such that the sum of both carries essentially the features of the known one. The method based on constant band areas, on the other hand, is most reliable in cases where the contributions of reactants and products have their strongest contributions in different spectral regions, such that the ratio $\alpha$ is sufficiently apart from unity and inverse for the two species.

In addition to the described methods, transient anisotropy measurements can be used for disentangling as well. For instance, the transient spectrum of Pe obtained at $500 \mathrm{ps}$ (fig. 2, upper panel) is composed out of ESA $(<18.5 \mathrm{kK}$ and $>20 \mathrm{kK})$, SE (19-23 kK) and ground state bleach $(>23 \mathrm{kK})$. The anisotropy on these data (fig. 2, lower panel) is featureless in the range $<17 \mathrm{kK}$ of the strong ESA. The pole around $18 \mathrm{kK}$ is due to the fact that the signal at magic angle crosses the zero line, whereas the composed anisotropy from ESA and SE does not. Above $19 \mathrm{kK}$ the measured anisotropy is surprisingly rich at first sight, stemming from the superposition of the ground state bleach, respectively the SE, both with anisotropy 0.4 on one side, and an ESA with different anisotropy on the other side. Note that the pronounced features in the anisotropy spectrum with $r>0.4$ are located where the SE and bleach signals pass through minima, so that the contribution from the ESA has the maximal relative weight there. The pure aspect of the signal-to-noise to be seen in fig. 2 strongly suggests that the contained information can be used for extracting the shape of the unknown ESA band. The information contained in the anisotropy spectrum can be exploited as follows. The idea follows a procedure developed for steady state spectra described by Valeur and Weber [7]. The signal at magic angle is given by

$$
\Delta A_{\text {tot }}=\Delta A_{\text {bleach }}+\Delta A_{\mathrm{ESA}}+\Delta A_{\mathrm{SE}}
$$




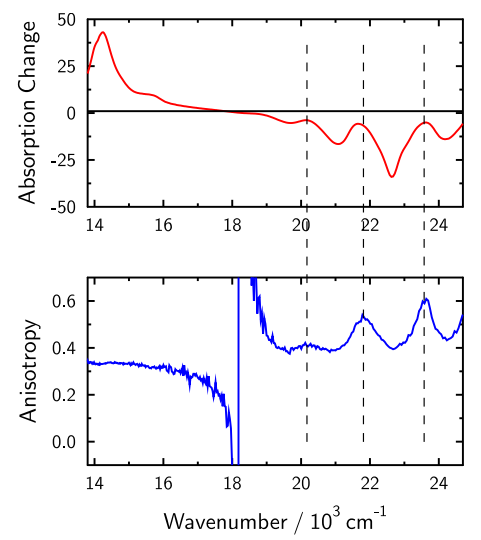

Figure 2: Transient absorption of perylene in acetonitrile at $500 \mathrm{fs}$ after photoexcitation at magic angle (upper panel), transient anisotropy at the same configuration (lower panel).

while the measured anisotropy can be written as

$$
R_{\mathrm{tot}}=\frac{\Delta A_{\text {bleach }} r_{\text {bleach }}+\Delta A_{\mathrm{ESA}} r_{\mathrm{ESA}}+\Delta A_{\mathrm{SE}} r_{\mathrm{SE}}}{R_{\mathrm{tot}}} .
$$

In addition to the steady state absorption spectrum the time resolved emission spectrum has to be determined using broadband timeresolved fluorescence up-conversion [8]. Combining both equations to eliminate the unknown spectrum of the ESA, $\Delta A_{\mathrm{ESA}}$, yields a nonlinear expression containing known spectra, the unknown anisotropy of the ESA, $r_{\mathrm{ESA}}$, and the scaling factors between the three experiments. Since this expression has to be valid for all recorded pixel values, it is largely over determined and can be fitted to the measured anisotropy. Fitting parameters are $r_{\mathrm{ESA}}$ and the scaling factors between the three experiments, TA, steady state absorption and time resolved fluorescence. A crucial precondition for this procedure to work is of course that all three experiments are photometrically matched. Future measurements will have to test the reliability of anisotropy as means for disentangling spectra under various experimental conditions.

Generally speaking, to determine an unknown spectral component, additional information is needed which is valid over the full spectral range of that component. Which is the method of choice is dictated by the actual molecular system under investigation. The optimal strategy has to be developed individually. Structured residuals in the fitting procedure of the anisotropy signal indicate further components being present which are not included in the model. When more than one unknown and overlapping signature is present, regularization methods like maximum entropy are at hand for further stabilizing the employed procedure.

The authors gratefully acknowledge financial support from the Swiss National Science Foundation, project No. 200020-165890 and grant IZK0Z2_170389, and the Narodowe Centrum Nauki (Poland) through grant SONATA bis No. 2013/10/E/ST4/00534.

\section{References}

[1] C. Ruckebusch, M. Sliwa, P. Pernot, A. de Juan, and R. Tauler, J. Photochem. Photobiol. C-Photochem. Rev. 13, 1 (2012).

[2] I. H. M. van Stokkum, D. S. Larsen, and R. van Grondelle, Biochim. Biophys. Acta - Bioenerg. 1657, 82 (2004).

[3] H. Marciniak and S. Lochbrunner, Chem. Phys. Lett. 609, 184 (2014).

[4] C. Slavov, H. Hartmann, and J. Wachtveitl, Anal. Chem. 87, 2328 (2015).

[5] G. Angulo, A. Rosspeintner, B. Lang, and E. Vauthey, submitted.

[6] R. Wilcken et al., J. Am. Chem. Soc. 140, 5311 (2018).

[7] B. Valeur and G. Weber, Photochem. Photobiol. 25, 441 (1977).

[8] X.-X. Zhang et al., Rev. Sci. Instrum. 82, 063108 (2011). 